life for many people. Increasingly it is recognised that negative disruptive impacts of the pandemic are not experienced equally and may exacerbate existing inequalities. People already suffering from psychological distress may have been especially vulnerable to disruptions. We investigated associations between pre-pandemic psychological distress and disruptions to healthcare, economic activity, and housing, and whether these associations were moderated by age, sex, ethnicity or education.

Methods Data were from 59,482 participants in 12 UK longitudinal adult population surveys with both pre-pandemic and COVID-19 surveys. Participants self-reported disruptions since the start of the pandemic to: healthcare (medication access, procedures, or appointments); economic activity (negative changes in employment, income or working hours); and housing (change of address or household composition). These were also combined into a cumulative measure indicating how many of these three domains had been disrupted. Logistic regression models were used within each study to estimate associations between pre-pandemic standardised psychological distress scores and disruption outcomes. Analyses were weighted for sampling design and attrition, and adjusted for age, sex, education, ethnicity, and UK country. Findings were synthesised using a random effects meta-analysis with restricted maximum likelihood. Effect modification by sex, education, ethnicity and age was assessed using group-difference tests during meta-analysis.

Results While exact prevalence varied between studies, prepandemic psychological distress was generally more common among women, ethnic minorities, younger age groups, and those with less education. One standard deviation higher psychological distress was associated with raised odds of health care disruptions (OR 1.40; 95\% CI: 1.29-1.51; Heterogeneity $\left.\mathrm{I}^{2}: 79.4 \%\right)$ and with experiencing disruptions in two or more of the three domains examined (OR 1.22; 95\% CI: 1.141.31 ; Heterogeneity $\mathrm{I}^{2}$ : $75.8 \%$ ), but not specifically with disruptions to economic activity (OR 1.03; 95\% CI: 0.95-1.13; Heterogeneity $\mathrm{I}^{2}$ : $89.5 \%$ ) or housing (OR 1.00; 95\% CI: 0.97-1.03; Heterogeneity $\left.\mathrm{I}^{2}: 0.0 \%\right)$. We did not find evidence of these associations differing by sex, ethnicity, education, or age group.

Conclusion Those suffering from psychological distress before the pandemic have been more likely to experience healthcare disruptions during the pandemic, and clusters of disruptions across multiple life domains. Individuals suffering from distress may need additional support to manage these disruptions, especially in relation to healthcare. Otherwise, considering psychological distress was already unequally distributed, the pandemic may exacerbate existing inequalities related to gender, ethnicity, education and age.

\section{OP66 HOW THE DESIGN AND NATURE OF GAMBLING MARKETING AFFECTS CONSUMERS: FINDINGS FROM A CONTENT ANALYSIS OF ADVERTISING AND IN-DEPTH INTERVIEWS WITH SPORTS BETTORS AND YOUNG PEOPLE DURING THE COVID-19 PANDEMIC IN THE UNITED KINGDOM}

Nathan Critchlow* ${ }^{1}$ Ashley Brown ${ }^{2} \mathrm{Craig}$ Donnachie ${ }^{1}$ Kate Hunt, ${ }^{2}$ Heather Ward ${ }^{1}$ Kathryn Angus. 'Institute for Social Marketing and Health, University of Stirling, Stirling, UK; ${ }^{2}$ School of Social and Political Sciences, University of Glasgow, Glasgow, UK

10.1136/jech-2021-SSMabstracts.66
Background Gambling advertising often contains a multitude of information about inducements and incentives, in addition to details of the specific gambling product marketed and other promotional features. Research suggests that consumers are more likely to misjudge the likelihood of winning or benefiting as the complexity of the gambling product offered increases. As part of a larger study concerning the impact of COVID-19 on gambling behaviours and marketing, we explored how gambling products and inducements are marketed in the United Kingdom (UK) and how inducements are received, and perceived, by gamblers.

Methods Two data sources are synthesised from the 'Betting and Gaming COVID-19 Impact Study'. An in-depth content analysis was conducted on a stratified random sample of gambling adverts $(n=200)$ from seven media activities in UK (March-May 2020). Coding captured information about the gamble promoted (e.g. suggested odds and wagers), inducements (e.g. new customer offers, free bets), and how Terms and Conditions (T\&Cs) were communicated. In-depth telephone interviews were conducted (July-November 2020) with sports bettors $(n=16)$ and young adults $(n=11)$ in the UK to explore experiences and practices related to gambling. Data were analysed thematically using the framework method.

Results Gambling adverts routinely featured complex information about the gambling products promoted and associated inducements. The design of adverts appeared carefully curated so that promotional and branding aspects had greater prominence than practical information about how inducements operated and eligibility to participate and benefit, if such T\&Cs appeared at all. In the interviews, participants perceived gambling advertising to be ubiquitous. While several participants underplayed the influence that gambling advertising and marketing had on their gambling activity, some explicitly said that the content had a negative impact on their gambling, including the adoption of novel and potentially 'riskier' gambling behaviours (e.g. online casino games). A few highlighted a lack of transparency in relation to T\&Cs underpinning inducements offered by gambling operators, which, in turn, compounded adverse gambling experiences.

Conclusion The way that gambling products and inducements are marketed in the UK is complex and likely to challenge comprehension by consumers. Bettors routinely recall awareness of, and engagement with, a variety of gambling marketing activities, and highlight concerns about the reach and impact that marketing may have, particularly on vulnerable groups such as young people and problem gamblers.

\section{Friday 17 September \\ Tobacco, $13.00-15.30$}

\section{OP67 WHEN MY WORKPLACE IS YOUR HOME: DOMICILIARY WORKERS' EXPOSURE TO SECOND-HAND TOBACCO SMOKE}

${ }^{1}$ Ruaraidh Dobson*, ${ }^{2}$ Evangelia Demou, ${ }^{1}$ Sean Semple. ${ }^{1}$ Institute for Social Marketing and Health, University of Stirling, Stirling, UK; ${ }^{2}$ MRC-CSO Social and Public Health Sciences Unit, University of Glasgow, Glasgow, UK

10.1136/jech-2021-SSMabstracts.67

Background Second-hand smoke (SHS) exposure at work is a cause of serious ill-health. Consequently, many countries have 